\title{
Left ventricular apical rotation is associated with mitral annular function in healthy subjects. Results from the three-dimensional speckle-tracking echocardiographic MAGYAR-Healthy Study
}

\author{
A. NEMES ${ }^{1 *}$ (®) Z. KOVÁCS ${ }^{2}$, Á. KORMÁNYOS ${ }^{1}$, P. DOMSIK $^{1}$, \\ A. KALAPOS ${ }^{1}$, Z. AJTAY ${ }^{3,4}$ and C. LENGYEL ${ }^{5}$
}

\footnotetext{
${ }^{1}$ 2nd Department of Medicine and Cardiology Centre, Medical Faculty, Albert Szent-Györgyi Clinical Center, University of Szeged, Szeged, Hungary

${ }^{2}$ Department of Cardiology, Szent Rókus Hospital, Baja, Hungary

${ }^{3}$ Vilmos Zsigmondy SPA Hospital, Harkány, Hungary

${ }^{4}$ Heart Institute, Medical School, University of Pécs, Pécs, Hungary

${ }^{5} 1$ st Department of Medicine, Medical Faculty, Albert Szent-Györgyi Clinical Center, University of Szeged, Szeged, Hungary
}

Received: February 19, 2019 • Accepted: December 9, 2019

Published online: April 23, 2020

(C) 2020 The Author(s)

\begin{abstract}
Introduction: Left ventricular (LV) twist is considered an essential part of LV function due to oppositely directed LV basal and apical rotations. Several factors could play a role in determining LV rotational mechanics in normal circumstances. This study aimed to investigate the relationship between LV rotational mechanics and mitral annular (MA) size and function in healthy subjects. Methods: The study comprised 118 healthy adult volunteers (mean age: $31.5 \pm 11.8$ years, 50 males). All subjects had undergone complete two-dimensional (2D) Doppler echocardiography and three-dimensional speckle-tracking echocardiography (3DSTE) at the same time by the same echocardiography equipment. Results: The normal mean LV apical and basal rotations proved to be $9.57 \pm 3.33$ and $-3.75 \pm 1.98^{\circ}$, respectively. LV apical rotation correlated with end-systolic MA diameter, area, perimeter, fractional area change, and fractional shortening, but did not correlate with any end-diastolic mitral annular morphologic parameters. The logistic
\end{abstract}

${ }^{*}$ Corresponding author. Semmelweis street 8, P.O. Box 427, Szeged, H-6725, Hungary. Tel: 36 62 545220; Fax: 36 62 544568, E-mail: nemes.attila@med.u-szeged.hu 
regression model identified MA fractional area change as an independent predictor of $\leq 6^{\circ}$ left ventricular apical rotation $(P<0.003)$. Conclusions: Correlations could be detected between apical LV rotation and end-systolic MA size and function, suggesting relationships between MA dimensions and function and LV rotational mechanics.

\section{KEYWORDS}

healthy, left ventricular, rotation, mitral annulus, three-dimensional, speckle-tracking, echocardiography

\section{INTRODUCTION}

Left ventricular (LV) twist is considered an essential part of LV function due to oppositely directed LV basal and apical rotation [8, 11]. Non-invasive techniques such as magnetic resonance imaging and three-dimensional (3D) speckle-tracking echocardiography (3DSTE) are useful to estimate LV twist dynamics in clinical settings [2, 7]. 3DSTE has been validated for the assessment of LV rotational mechanics [16], and normal ranges for 3DSTE-derived parameters in pediatric [15] and adult [4, 12] populations have also been demonstrated. The exact assessment of mitral annular (MA) size and function in routine clinical practice is also essential due to their changes in different pathological states. 3D echocardiographic evaluation of morphologic and functional aspects of MA has been demonstrated and validated $[1,9]$. However, little is known about their relationship with LV rotational mechanics even in normal circumstances. Therefore, we used 3DSTE to investigate the relationship between LV rotational mechanics and MA size and function in healthy subjects.

\section{METHODS}

\section{Subjects}

The study comprised 118 healthy adult volunteers (mean age: $31.5 \pm 11.8$ years, 50 males) who were primarily recruited on voluntary basis. None of them had risk factors, known diseases or other conditions or received medications which could theoretically affect the results. All subjects had undergone complete two-dimensional (2D) Doppler echocardiography and 3DSTE at the same time by the same echocardiography equipment. The results presented are a part of the Motion Analysis of the heart and Great vessels bY three-dimensionAl speckle-tRacking echocardiography in Healthy subjects (MAGYAR-Healthy) Study, which was organized in the Cardiology Center of University of Szeged. This study aimed to assess, among others, the clinical significance of 3DSTE-derived parameters in healthy subjects ("magyar" means "Hungarian" in Hungarian language). Informed consent was obtained from all subjects, and the Ethics Committee of the University of Szeged, Hungary approved the study.

\section{Two-dimensional echocardiography}

2D Doppler echocardiography was used in all subjects with a commercially available ultrasonic device (Toshiba Artida ${ }^{\mathrm{TM}}$; Toshiba Medical Systems, Tokyo, Japan) using a PST-30SBP (1-5 $\mathrm{MHz}$ ) phased-array transducer to measure LV diameters and volumes respecting the cardiac 
cycle and left atrial diameter [5]. Visual grading and Doppler assessments were used to exclude valvular abnormalities.

\section{DSTE-derived data acquisition and quantitative analysis}

The same Toshiba Artida ${ }^{\mathrm{TM}}$ ultrasound system was used with a fully sampled PST-25SX matrixarray transducer (Toshiba Medical Systems, Tokyo, Japan) with 3D capability. 3D pyramidal data acquisitions were performed with regard to recent practices. To reach optimal temporal and spatial resolution, depth and angle were adjusted. Six consecutive cardiac cycles were used to acquire $6 \mathrm{R}$-wave-triggered LV subvolumes during one breath-hold, from which a pyramidshaped 3D full volume was formed by the software. 3D Wall Motion Tracking software version 2.7 (Toshiba Artida ${ }^{\mathrm{TM}}$; Toshiba Medical Systems, Tokyo, Japan) was used for quantitative analysis. The mean volume rate was $24 \pm 3$ vps in our practice.

\section{DSTE-derived LV rotational parameters}

Three short-axis views at different LV levels and the apical two- (AP2CH) and four-chamber $(\mathrm{AP} 4 \mathrm{CH})$ views were selected automatically from the 3D echocardiographic pyramidal dataset at end-diastole by the software [2]. On the apical $\mathrm{AP} 2 \mathrm{CH}$ and $\mathrm{AP} 4 \mathrm{CH}$ views, three points were selected for $3 \mathrm{D}$ reconstruction of the endocardial surface: two points were selected at the edges of the mitral valve and one was selected at the apex. Subsequently, the endocardial surface was manually adjusted in all apical and short-axis views. Then, the $3 \mathrm{D}$ endocardial surface was automatically reconstructed and tracked in $3 \mathrm{D}$ space throughout the cardiac cycle. Curves for quantification of LV apical and basal rotations were generated by the software (Fig. 1).

\section{DSTE-derived MA measurements}

A recently demonstrated simple method was used for MA assessments. Briefly, to calculate morphological MA parameters at end-diastole and end-systole, the short-axis reconstructed MA view was positioned at the level of MA, where $\mathrm{AP} 2 \mathrm{CH}$ and $\mathrm{AP} 4 \mathrm{CH}$ views helped to find optimal endpoints of the MA (Fig. 2) [9]:

\section{MA morphologic parameters.}

- MA short or septolateral diameter, defined as the perpendicular line drawn from the peak of MA curvature to the middle of the straight MA border both at end-diastole (just before mitral valve closure) and end-systole (just before mitral valve opening),

- MA area, measured at end-diastole and at end-systole by planimetry,

- MA perimeter, measured at end-diastole and at end-systole by planimetry,

\section{MA functional parameters.}

- MA fractional shortening (MAFS), defined as: (end-diastolic MA diameter - end-systolic MA diameter $) /($ end-diastolic MA diameter $\times 100)$, and

- MA fractional area change (MAFAC), defined as: (end-diastolic MA area - end-systolic MA area $) /($ end-diastolic MA area $\times 100)$ 


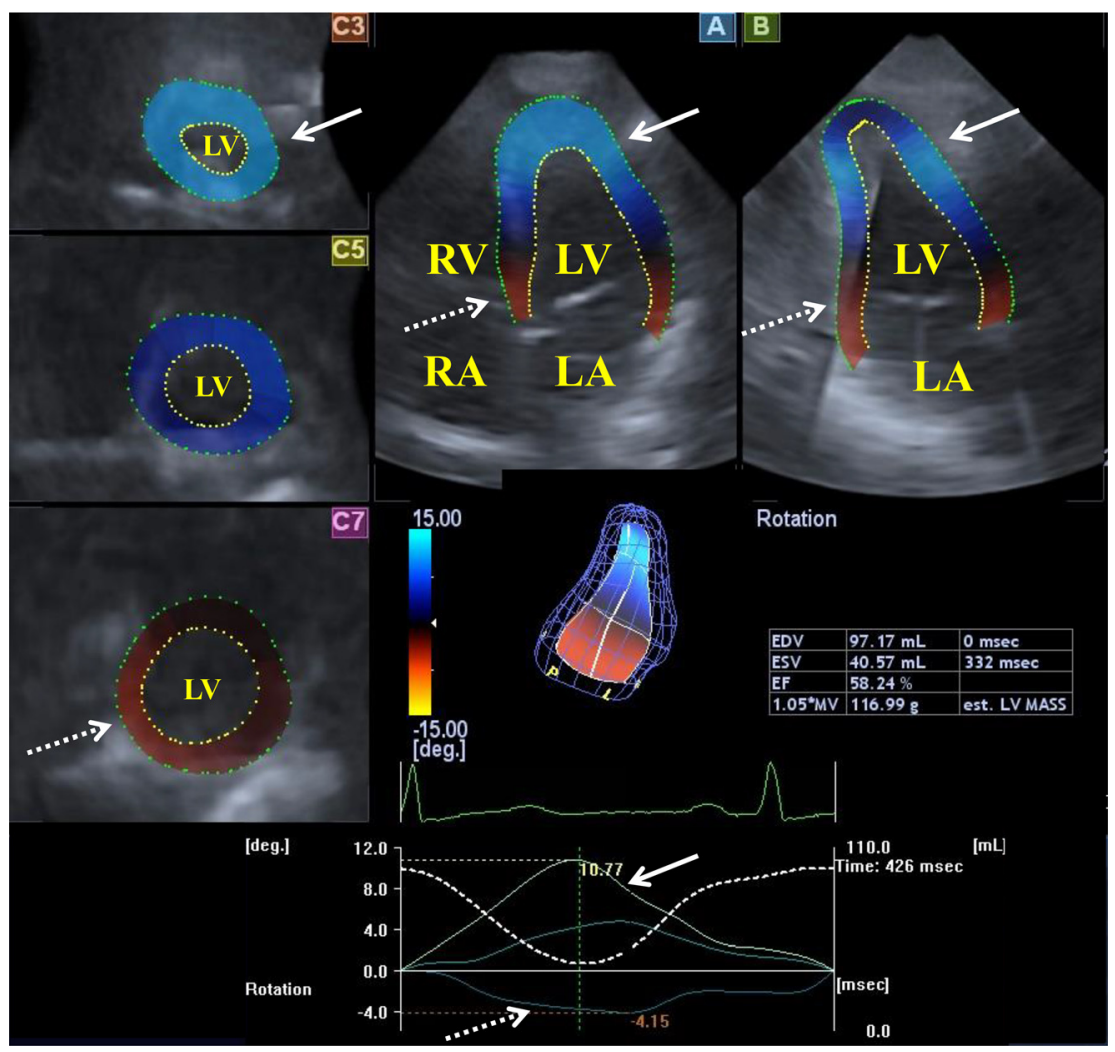

Figure 1. Apical two- and four-chamber views and 3 short axis views at different left ventricular levels in a healthy woman are presented. Global and apical (white arrow), midventricular and basal (dashed arrow) regional left ventricular rotation curves detected by three-dimensional speckle-tracking echocardiography are demonstrated. The adequate rotational directions are: counterclockwise motion of the left ventricular apex expressed with positive values (white arrow, blue colored) and clockwise rotation of the left ventricular base with negative values (dashed arrow, red colored).Abbreviations: $\mathrm{LA}=$ left atrium, $\mathrm{LV}=$ left ventricle, $\mathrm{RA}=$ right atrium, $\mathrm{RV}=$ right ventricle

\section{Statistical analysis}

Quantitative data were expressed as mean \pm standard deviation. For all analyses, two-sided $P<$ 0.05 was defined as statistical significance. Pearson's coefficient was calculated to examine correlations between parameters featuring MA morphology and function and LV rotational mechanics. To establish the prognostic power of MA morphologic and functional parameters in predicting reduced LV apical and basal rotations, receiver operator curves were constructed and the area under the curve was reported with sensitivity and specificity values. Univariate analysis of factors (end-systolic and end-diastolic MA diameter, area, perimeter, fractional area change, and fractional shortening) was performed. Significant variables from univariable analysis $(P<$ $0.10)$ were integrated into multivariable analysis for assessment of independent predictors of $\leq 6^{\circ}$ 


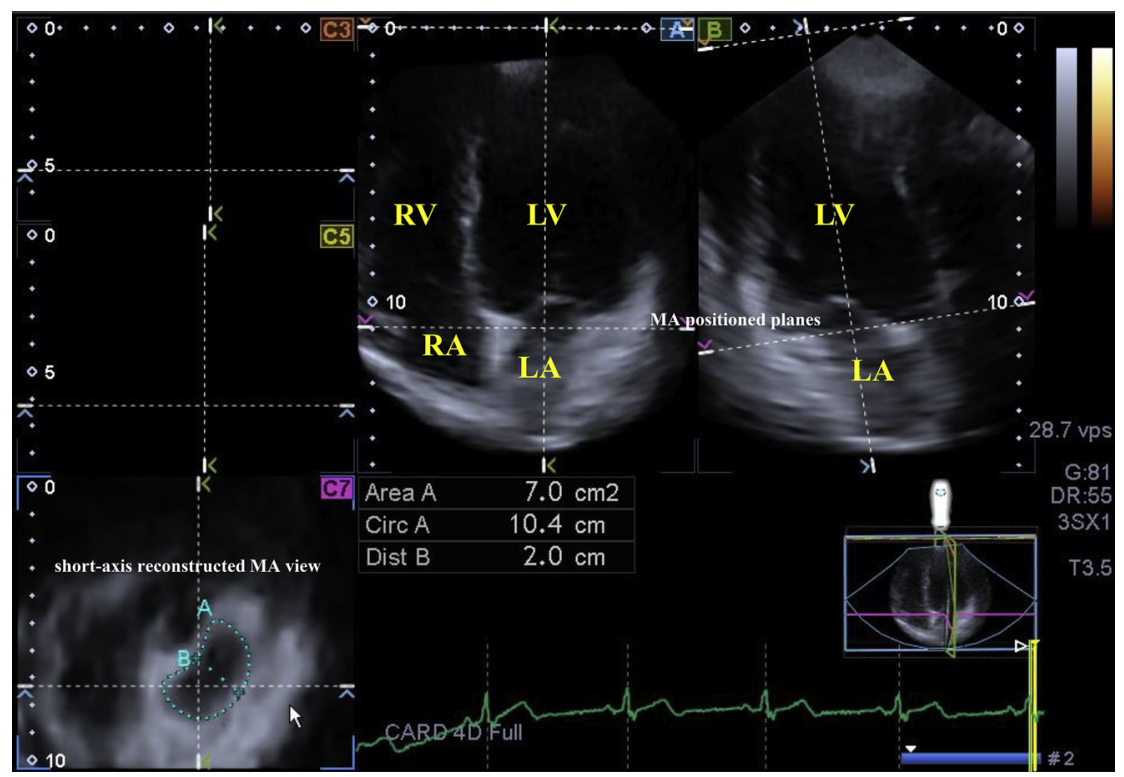

Figure 2. Images from three-dimensional full-volume dataset showing left ventricle in a healthy case: (A) apical four-chamber view, (B) apical two-chamber view and a cross-sectional view at the level of the mitral annulus (C7) optimized on apical four- and two-chamber views.Abbreviations: Area = mitral annular area, Circ $=$ mitral annular perimeter, Dist $=$ mitral annular diameter, $L A=$ left atrium, $L V=$ left ventricle, $\mathrm{RA}=$ right atrium, $\mathrm{RV}=$ right ventricle

LV apical rotation and $\leq-2^{\circ} \mathrm{LV}$ basal rotation (approximated mean minus standard deviation). Commercially available MedCalc software was used for statistical calculations (MedCalc, Mariakerke, Belgium).

\section{RESULTS}

\section{Clinical data}

Routine 2D Doppler echocardiography showed normal results (left atrium: $38.7 \pm 2.7 \mathrm{~mm}$, LV end-systolic diameter: $48.7 \pm 2.5 \mathrm{~mm}$, LV end-diastolic diameter: $32.6 \pm 2.6 \mathrm{~mm}$, interventricular septum: $9.0 \pm 0.9 \mathrm{~mm}, \mathrm{LV}$ posterior wall: $8.9 \pm 0.8 \mathrm{~mm}$, LV ejection fraction: $65.6 \pm$ $2.9 \%$ ). None of the healthy subjects had grade $\geq 1$ valvular regurgitation or significant valvular stenosis. 3DSTE data are presented in Table 1.

\section{The relationship between MA size and function and LV apical rotation}

The normal mean LV apical rotation proved to be $9.57 \pm 3.33^{\circ}$. Patients with higher than average LV apical rotation had lower systolic MA diameter, area and perimeter, but MA functional properties (MAFAC and MAFS) proved to be increased (Table 1). Moreover, LV 
Table 1. Three-dimensional speckle-tracking echocardiographic data of healthy subjects and their relationship to left ventricular rotational parameters

\begin{tabular}{|c|c|c|c|c|c|}
\hline & $\begin{array}{c}\text { All } \\
(\mathrm{n}=118)\end{array}$ & $\begin{array}{l}\mathrm{LV} \text { apical } \\
\text { rotation } \leq 9.57^{\circ} \\
(\mathrm{n}=53)\end{array}$ & $\begin{array}{l}\mathrm{LV} \text { apical } \\
\text { rotation }>9.57^{\circ} \\
\quad(\mathrm{n}=65)\end{array}$ & $\begin{array}{l}\mathrm{LV} \text { basal } \\
\text { rotation } \leq 3.75^{\circ} \\
(\mathrm{n}=49)\end{array}$ & $\begin{array}{c}\mathrm{LV} \text { basal } \\
\text { rotation }>3.75^{\circ} \\
\quad(\mathrm{n}=69)\end{array}$ \\
\hline \multicolumn{6}{|l|}{ LV volumetric data } \\
\hline $\begin{array}{l}\text { End-diastolic } \\
\text { LV volume (mL) }\end{array}$ & $86.04 \pm 24.96$ & $88.70 \pm 24.33$ & $84.23 \pm 25.89$ & $86.78 \pm 24.07$ & $86.13 \pm 25.91$ \\
\hline $\begin{array}{l}\text { End-systolic } \\
\text { LV volume }(\mathrm{mL})\end{array}$ & $36.60 \pm 10.97$ & $37.91 \pm 10.51$ & $35.07 \pm 11.65$ & $35.69 \pm 10.83$ & $37.24 \pm 11.19$ \\
\hline LV ejection fraction (\%) & $57.98 \pm 5.15$ & $57.79 \pm 5.40$ & $58.44 \pm 5.13$ & $58.97 \pm 4.95$ & $57.28 \pm 5.25$ \\
\hline \multicolumn{6}{|l|}{ LV rotational parameters } \\
\hline LV apical rotation (degree) & $9.57 \pm 3.33$ & $7.38 \pm 1.90$ & $12.42 \pm 2.42^{\star}$ & $8.86 \pm 3.26$ & $10.08 \pm 3.34 \dagger$ \\
\hline LV basal rotation (degree) & $-3.75 \pm 1.98$ & $-4.05 \pm 1.90$ & $-3.36 \pm 1.94^{*}$ & $-5.56 \pm 1.67$ & $-2.47 \pm 0.90 \dagger$ \\
\hline \multicolumn{6}{|l|}{ MA parameters } \\
\hline $\begin{array}{l}\text { End-diastolic MA diameter } \\
\quad(\mathrm{cm})\end{array}$ & $2.44 \pm 0.45$ & $2.44 \pm 0.47$ & $2.44 \pm 0.47$ & $2.45 \pm 0.47$ & $2.42 \pm 0.45$ \\
\hline End-diastolic MA area $\left(\mathrm{cm}^{2}\right)$ & $7.14 \pm 2.10$ & $7.04 \pm 2.17$ & $7.22 \pm 2.05$ & $7.14 \pm 1.98$ & $7.14 \pm 2.20$ \\
\hline $\begin{array}{l}\text { End-diastolic MA } \\
\text { perimeter }(\mathrm{cm})\end{array}$ & $10.10 \pm 1.41$ & $9.99 \pm 1.43$ & $10.18 \pm 1.39$ & $10.08 \pm 1.33$ & $10.10 \pm 1.48$ \\
\hline $\begin{array}{l}\text { End-systolic MA diameter } \\
(\mathrm{cm})\end{array}$ & $1.62 \pm 0.38$ & $1.73 \pm 0.36$ & $1.48 \pm 0.37^{*}$ & $1.62 \pm 0.38$ & $1.63 \pm 0.39$ \\
\hline End-systolic MA area $\left(\mathrm{cm}^{2}\right)$ & $3.47 \pm 1.18$ & $3.73 \pm 1.19$ & $3.11 \pm 1.08^{\star}$ & $3.54 \pm 1.01$ & $3.42 \pm 1.30$ \\
\hline $\begin{array}{l}\text { End-systolic MA } \\
\text { perimeter }(\mathrm{cm})\end{array}$ & $7.12 \pm 1.15$ & $7.35 \pm 1.11$ & $6.80 \pm 1.13^{*}$ & $7.26 \pm 0.95$ & $7.02 \pm 1.28$ \\
\hline $\begin{array}{l}\text { MA fractional } \\
\text { area change (\%) }\end{array}$ & $49.80 \pm 14.82$ & $44.99 \pm 14.77$ & $55.98 \pm 12.20^{*}$ & $49.08 \pm 12.28$ & $50.31 \pm 16.55$ \\
\hline $\begin{array}{l}\text { MA fractional } \\
\text { shortening (\%) }\end{array}$ & $32.29 \pm 15.26$ & $28.19 \pm 14.13$ & $38.06 \pm 15.26^{*}$ & $32.96 \pm 15.10$ & $31.81 \pm 15.57$ \\
\hline
\end{tabular}



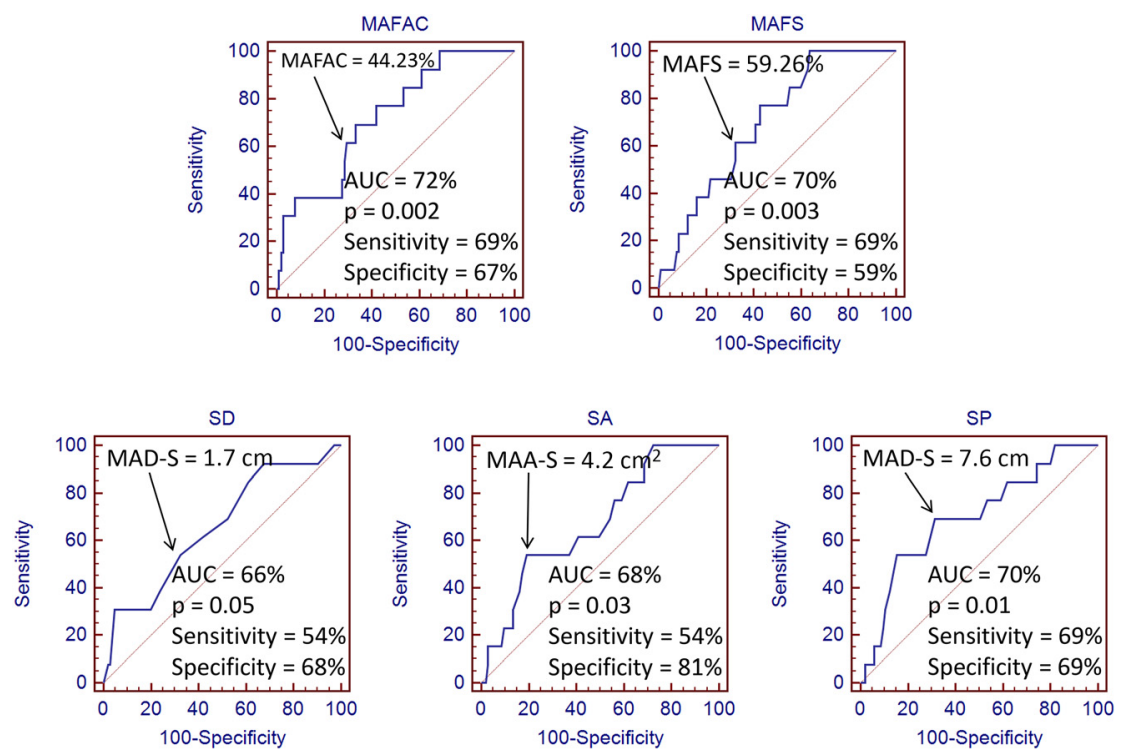

Figure 3. Receiver operating characteristic analyses illustrating the diagnostic accuracy of MA fractional area change (MAFAC), MA fractional shortening (MAFS), end-systolic MA diameter (MAD-S), endsystolic MA area (MAA-S) and end-systolic MA perimeter (MAP-S) in predicting reduced $\left(\leq 6^{\circ}\right)$ left ventricular apical rotation

apical rotation correlated with end-systolic MA diameter $(\mathrm{r}=-0.320, \mathrm{p}=0.0004)$, endsystolic MA area $(\mathrm{r}=-0.309, \mathrm{p}=0.0007)$, end-systolic MA perimeter $(\mathrm{r}=-0.314, \mathrm{p}=$ $0.0005)$, MAFAC $(\mathrm{r}=0.439, \mathrm{p}<0.0001)$ and MAFS $(\mathrm{r}=0.350, \mathrm{p}=0.0001)$, but did not correlate with any end-diastolic MA morphologic parameters. Less than $6^{\circ} \mathrm{LV}$ apical rotation was considered to be reduced. The diagnostic accuracy of MAFAC, MAFS, end-systolic MA diameter, area and perimeter in predicting $\leq 6^{\circ} \mathrm{LV}$ apical rotation is demonstrated in Fig. 3. The logistic regression model identified MA fractional area change as an independent predictor of $\leq 6^{\circ} \mathrm{LV}$ apical rotation $(P<0.003)$.

\section{The relationship between MA size and function and LV basal rotation}

The normal mean LV basal rotation was considered to be $-3.75 \pm 1.98^{\circ}$. No relationship could be detected between LV basal rotation and the above mentioned 3DSTE parameters. LV basal rotation did not correlate with any MA morphologic or functional parameter. If less than $-2^{\circ}$ $\mathrm{LV}$ basal rotation was considered to be reduced, none of the MA parameters was found to predict reduced LV basal rotation.

\section{DISCUSSION}

The LV ejection is not simply the result of the contraction of myocardial fibers, but is significantly affected by the twisting motion of the LV. In normal circumstances, LV base 
rotates in clockwise direction and $\mathrm{LV}$ apex rotates in counterclockwise direction resulting in a towel-wringing motion called LV twist [6]. According to Nakatani, LV twist is significantly governed by the degree of contraction and relaxation of the myocardium, the balance between contraction of the subendocardium and subepicardium and the orientation of the myocardial fibers [6]. However, several physiologic determinants of LV twist are known including age, changes of loading and contractility, and the effect of exercise. Moreover, myocardial structural abnormalities could also have an impact on LV twist [10]. Newer echocardiographic techniques including 3DSTE are demonstrated to be feasible for non-invasive quantification of LV rotational mechanics [16]. Due to recent guidelines, 2D speckle-tracking echocardiographic evaluation of LV rotational mechanics is not suggested, only its 3DSTE evaluation is preferred [14].

The MA is a crucial and dynamic structure and is a tightly coupled component of the mitral valve-left atrium-LV complex that aids in effective and efficient valve closure and unimpeded LV filling [13]. 3D echocardiography allows detailed assessment of MA measures respecting the cardiac cycle [1,9]. In normal hearts, LV twist mechanics are load-dependent [10], the load is theoretized to be affected by MA size and function. Therefore, the present study aimed to assess whether MA morphologic and functional properties affect and predict LV rotational features in healthy circumstances. In recent studies, different LV-related pathologic states involving the mitral valve were found to be related to LV rotational mechanics. For instance, peak LV twist, peak LV twist rate, and LV torsion were increased in the presence of MA calcification, and these parameters correlated with the presence and the severity of MA calcification [3]. In another study, impaired basal rotational mechanics occurring after an inferior-posterior myocardial infarction were found to be associated with increased mitral regurgitation [17]. The importance of the present study is that relationships could be confirmed between LV apical rotation and MA end-systolic morphology and MA function even in healthy subjects without calcification or regurgitation. Moreover, end-systolic MA measures and MA functional properties showed prognostic impact in predicting reduced $\left(\leq 6^{\circ}\right) \mathrm{LV}$ apical rotation, and MAFAC was found to be an independent predictor of reduced LV apical rotation.

\section{LIMITATION SECTION}

Several important issues arose during assessments. The most important ones are listed below:

- A young population of healthy subjects was examined, and the conclusions may not translate into healthy elderly, which should be considered as a significant limitation of this study.

- 3DSTE is a relatively new methodology with low spatial and temporal resolution. These technical limitations could affect the results, and should be considered when interpreting the results.

- LV strain assessments and volumetric and functional characterization of heart chambers other than LV were not aimed in this study.

- Only in-plane MA motion and function were analyzed in this study. Although analysis of the MA movement along the LV long-axis is also possible [7], it was not aimed to be measured and compared to other parameters in this particular study.

- Single vendor measurements were performed, which could be considered as a potential limitation. 


\section{CONCLUSIONS}

Correlations could be detected between apical left ventricular rotation and end-systolic MA size and function, suggesting relationships between MA dimensions and function and LV rotational mechanics.

Ethical standards: The authors assert that all procedures contributing to this work comply with the ethical standards of the relevant national guidelines on human experimentation and with the Helsinki Declaration of 1975, as revised in 2008, and has been approved by the institutional committee of the University of Szeged.

Financial support: This work was supported by a grant from the National Research, Development and Innovation Office (GINOP-2.3.2-15-2016-00047).

Conflict of interest: None.

\section{REFERENCES}

1. Anwar AM, Soliman OI, Nemes A, Germans T, Krenning BJ, Geleijnse ML, et al. Assessment of mitral annulus size and function by real-time 3-dimensional echocardiography in cardiomyopathy: comparison with magnetic resonance imaging. J Am Soc Echocardiogr. 2007; 20: 941-8.

2. Biswas M, Sudhakar S, Nanda NC, Buckberg G, Pradhan M, Roomi AU, et al. Two- and three-dimensional speckle tracking echocardiography: clinical applications and future directions. Echocardiography 2013; 30: 88-105.

3. Gökdeniz T, Boyaci F, Hatem E, Bektaş H, Kalaycıŏlu E, Gürsoy MO, et al. Association of mitral annular calcification with left ventricular mechanics: a speckle tracking study. Echocardiography 2015; 32: 1374-83.

4. Kaku K, Takeuchi M, Tsang W, Takigiku K, Yasukochi S, Patel AR, et al. Age-related normal range of left ventricular strain and torsion using three-dimensional speckle-tracking echocardiography. J Am Soc Echocardiogr. 2014; 27: 55-64.

5. Lang RM, Badano LP, Mor-Avi V, Afilalo J, Armstrong A, Ernande L, et al. Recommendations for cardiac chamber quantification by echocardiography in adults: an update from the American Society of Cardiology and European Association of Cardiovascular Imaging. J Am Soc Echocardiogr. 2015; 28: 1-39.e14.

6. Nakatani S. Left ventricular rotation and twist: why should we learn? J Cardiovasc Ultrasound. 2011; 19: 1-6.

7. Nemes A, Forster T. Recent echocardiographic examination of the left ventricle - from M-mode to 3D speckle-tracking imaging. Orv Hetil. 2015; 156: 1723-40.

8. Nemes A, Kalapos A, Domsik P, Forster T. Left ventricular rotation and twist of the heart. Clarification of some concepts. Orv Hetil. 2012; 153: 1547-51.

9. Nemes A, Piros GÁ, Domsik P, Kalapos A, Lengyel C, Várkonyi TT, et al. Changes in mitral annular morphology and function in young patients with type 1 diabetes mellitus-results from the three-dimensional speckle tracking echocardiographic MAGYAR-path study. Quant Imaging Med Surg. 2015; 5: 815-21.

10. Omar AM, Vallabhajosyula S, Sengupta PP. Left ventricular twist and torsion: research observations and clinical applications. Circ Cardiovasc Imaging. 2015; 8: e003029.

11. Sengupta PP, Tajik AJ, Chandrasekaran K, Khandheria BK. Twist mechanics of the left ventricle: principles and application. JACC Cardiovasc Imaging. 2008; 1: 366-76. 
12. Tavakoli V, Sahba N. Assessment of age-related changes in left ventricular twist by 3-dimensional speckletracking echocardiography. J Ultrasound Med. 2013; 32: 1435-41.

13. Timek TA, Miller DC. Experimental and clinical assessment of mitral annular area and dynamics: what are we actually measuring? Ann Thorac Surg. 2001; 72: 966-74.

14. Voigt JU, Pedrizzetti G, Lysyansky P, Marwick TH, Houle H, Baumann R, et al. Definitions for a common standard for 2D speckle tracking echocardiography: consensus document of the EACVI/ASE/Industry Task Force to standardize deformation imaging. Eur Heart J Cardiovasc Imaging. 2015; 16: 1-11.

15. Zhang L, Zhang J, Han W, Gao J, He L, Yang Y, et al. Three-dimensional rotation, twist and torsion analyses using real-time 3D speckle tracking imaging: feasibility, reproducibility, and normal ranges in pediatric population. PLoS One 2016; 11: e0158679.

16. Zhou Z, Ashraf M, Hu D, Dai X, Xu Y, Kenny B, et al. Three-dimensional speckle-tracking imaging for left ventricular rotation measurement: an in vitro validation study. J Ultrasound Med. 2010; 29: 903-9.

17. Zito C, Cusmà-Piccione M, Oreto L, Tripepi S, Mohammed M, Di Bella G, et al. In patients with postinfarction left ventricular dysfunction, how does impaired basal rotation affect chronic ischemic mitral regurgitation? J Am Soc Echocardiogr. 2013; 26: 1118-29.

Open Access statement. This is an open-access article distributed under the terms of the Creative Commons Attribution 4.0 International License (https://creativecommons.org/licenses/by/4.0/), which permits unrestricted use, distribution, and reproduction in any medium, provided the original author and source are credited, a link to the CC License is provided, and changes - if any - are indicated. (SID_1) 